Pacific Journal of Mathematics

DERIVATIONS OF QUASITRIANGULAR ALGEBRAS 


\title{
DERIVATIONS OF QUASITRIANGULAR ALGEBRAS
}

\author{
BRUCE H. WAGNER
}

\begin{abstract}
If $\mathscr{Q}$ is a quasitriangular algebra, i.e., a compact perturbation of a nest algebra, then every automorphism of $Q$ close to the identity is inner. As a consequence, every derivation on $Q$ is inner.
\end{abstract}

Introduction. The derivation problem for nest algebras was solved by Christensen in [3], where it was shown that every derivation on a nest algebra is inner. The corresponding problem for quasitriangular algebras is different since these algebras are not weakly closed and since the nest which determines the algebra is not unique. The first result was obtained by Christensen and Peligrad [4] for the case of any nest which consists of an increasing sequence of finite rank projections which converges strongly to the identity operator. It was shown that every derivation on the associated quasitriangular algebra is inner. The proof used the fact that every automorphism on such an algebra is inner, proved by Plastiras in [16]. In [18], the author generalized these results for all quasitriangular algebras which have a determining nest of order type the extended natural numbers, the extended integers, or the unit interval $[0,1]$. In the latter case, Andersen's theorem (Theorem 3.5.5 of [1]) was crucial. The two former cases were proved by fairly direct methods, the basis of which were a preliminary result of Andersen (Corollary 1.4.3 of [1]) and a theorem of Davidson (Theorem 1.1 of [5]).

Recent important results of Larson [15] and Davidson [8] have made it possible to study automorphisms and derivations of arbitrary quasitriangular algebras, instead of using the case-by-case analysis of [18]. The main tools we use for this purpose are Theorems 1.3 and 1.5 below, due to Davidson [8]. The main result (Theorem 2.1) is that any automorphism $\alpha$ of a quasitriangular algebra with $\|\alpha-i d\|<\frac{1}{2}$ must be inner. It then follows easily (Theorem 2.2) that every derivation on a quasitriangular algebra is inner, thus answering a question posed by several people (see [9, $\S 3]$ and $[12, \S 3])$.

Other problems concerning quasitriangular algebras have been studied in [2], [6], [7], and [11], in addition to the references cited above. In particular, the results of [11] (Theorems 1.1 and 1.2 below) play an important role in our analysis.

Finally, we wish to thank William B. Arveson for his encouragement and helpful advice. 
1. Preliminaries. Throughout this paper, $\mathscr{H}$ will be a complex separable infinite-dimensional Hilbert space. The set of bounded operators on $\mathcal{H}$ is denoted by $\mathcal{L}(\mathcal{H})$ and the set of compact operators is denoted by $\mathscr{K}$. All operators discussed will be bounded, and all projections will be self-adjoint.

A nest is a family of projections in $\mathcal{L}(\mathcal{H})$ which is linearly ordered (by range inclusion), contains 0 and $I$, and is closed in the strong operator topology. A nest equipped with the strong operator topology forms a compact separable complete metric space. Given a nest $\mathscr{P}$, let alg $\mathscr{P}=\{T$ $\in \mathcal{L}(\mathcal{H}): P^{\perp} T P=0$ for all $\left.P \in \mathcal{P}\right\}$. alg $\mathcal{P}$ is the nest algebra associated with $\mathcal{P}$, and an operator $T \in$ alg $\mathcal{P}$ is said to be triangular with respect to $\mathscr{P}$. alg $\mathscr{P}$ is closed in the weak operator topology. Let $Q T(\mathscr{P})=\operatorname{alg} \mathscr{P}+\mathscr{K}$. $Q T(\mathscr{P})$ is the quasitriangular algebra associated with $\mathscr{P}$, and an operator $T \in Q T(\mathscr{P})$ is said to be quasitriangular relative to $\mathscr{P}$. The following two results concerning $Q T(\mathcal{P})$ were proved by Fall, Arveson, and Muhly in [11].

THEOREM 1.1. For any nest $\mathscr{P}, Q T(\mathcal{P})$ is a norm-closed algebra.

THEOREM 1.2. If $\mathcal{P}$ is a nest, then $Q T(\mathcal{P})=\{T \in \mathcal{L}(\mathcal{H})$ :

(i) $P^{\perp} T P \in \mathscr{K}$ for all $P \in \mathscr{P}$ and

(ii) the function $P \in \mathcal{P} \rightarrow P^{\perp} T P \in \mathcal{K}$ is strong-norm continuous (i.e., the function is continuous with respect to the strong operator topology on $\mathcal{P}$ and the norm topology on $\mathscr{K}$ ) \}.

Let $\Re$ be a Banach algebra. A derivation $\delta: \Re \rightarrow \Re$ is a linear function which has the property that $\delta(S T)=\delta(S) T+S \delta(T)$ for all $S, T \in \mathscr{B}$. We denote $\delta$ by ad $X$ if $\delta(S)=X S-S X$ for some $X \in \mathcal{L}(\mathcal{F C})$. $\delta$ is inner if $\delta=\operatorname{ad} X$ for some $X \in \mathscr{B}$. If $\alpha$ is an automorphism of $\mathscr{B}$, then we denote $\alpha$ by Ad $A$ if $\alpha(S)=A S A^{-1}$ for some invertible $A \in \mathcal{L}(\mathcal{H}) . \alpha$ is inner if $\alpha=\operatorname{Ad} A$ with $A, A^{-1} \in \mathscr{B}$. If $\delta$ is a continuous derivation, then $\delta$ is the infinitesimal generator of the uniformly continuous one-parameter automorphism group $\{\exp (t \delta): t \in \mathbf{R}\}$. If $\delta=\operatorname{ad} X$, then $\exp (t \delta)=$ $\operatorname{Ad}(\exp (t X))$.

Let $\mathcal{P}$ be a nest. An interval of $\mathscr{P}(\mathcal{P}$-interval, semi-invariant projection) is a projection $E=P^{\prime}-P$, with $P, P^{\prime} \in \mathcal{P}$ and $P<P^{\prime} . P$ and $P^{\prime}$ are called the lower and upper endpoints of $E$, respectively. It is easy to see that the endpoints of an interval are unique. An atom of $\mathscr{P}$ is a minimal $\mathscr{P}$-interval (or equivalently, a minimal projection in $\mathcal{P}^{\prime \prime}$, the double commutant of $\mathcal{P}$ ). $\mathscr{P}$ is continuous, or non-atomic, if it has no atoms. 
Two nests $\mathscr{P}$ and $\mathcal{Q}$ are unitarily equivalent if there is a unitary operator $U$ such that $2=\left\{U P U^{*}: P \in \mathscr{P}\right\}$. In this case, alg $\mathcal{Z}=$ $U(\operatorname{alg} \mathcal{P}) U^{*}$. In [10], Erdos completely analyzed the unitary invariants for nests. Two nests $\mathcal{P}$ and $\mathcal{Q}$ are similar if there is an invertible operator $A$ such that $\{Q \mathcal{H}: Q \in \mathcal{Q}\}=\{A P \mathcal{H}: P \in \mathcal{P}\}$. In this case, alg $\mathcal{Q}=$ $A(\operatorname{alg} \mathcal{P}) A^{-1}$, so $\operatorname{Ad} A$ is an isomorphism of alg $\mathcal{P}$ onto alg $\mathcal{Q}$, and it extends to an isomorphism of $Q T(\mathcal{P})$ onto $Q T(\mathcal{2})$. Given an order isomorphism $\theta: \mathscr{P} \rightarrow \mathcal{Q}$, we say that $\theta$ is implemented by $A$ if $A$ is an invertible operator and $\theta(P) \mathcal{H}=A P \mathcal{H}$ for all $P \in \mathscr{P} . \theta$ is dimension-preserving if $\operatorname{dim}\left(\theta\left(P^{\prime}\right)-\theta(P)\right) \mathcal{H}=\operatorname{dim}\left(P^{\prime}-P\right) \mathcal{H}$ for all $P, P^{\prime} \in \mathcal{P}$ with $P<P^{\prime}$. In [8], Davidson completely analyzed the similarity invariants for nests. Since we will need this result, we record it in the following theorem:

TheOREM 1.3. (Davidson [8, Theorem 5.1]). Two nests $\mathcal{P}$ and $\mathcal{Q}$ are similar if and only if there is a dimension-preserving order isomorphism $\theta$ of $\mathcal{P}$ onto 2 . Moreover, any dimension-preserving order isomorphism of $\mathcal{P}$ onto 2 can be implemented by an invertible operator which is an arbitrarily small compact perturbation of a unitary operator.

Theorem 1.3 has an interesting corollary (Corollary 1.4 below) which is related to the factorization property. A nest $\mathscr{P}$ is said to have the factorization property if, for every invertible operator $A$, there exists a unitary operator $U$ such that $A=U S$ for some $S \in(\operatorname{alg} \mathscr{P}) \cap(\operatorname{alg} \mathscr{P})^{-1}$. (An equivalent statement is that for every invertible operator $A$, there is a unitary operator $U$ such that $A P \mathcal{H}=U P \mathcal{H}$ for all $P \in \mathscr{P}$ ). In [15], Larson made a complete analysis of the factorization property. He showed that $\mathcal{P}$ has the factorization property if and only if $\mathcal{P}$ is countable, i.e., if $\mathscr{P}$ is uncountable, there is always some operator $A$ for which factorization fails. If we replace alg $\mathscr{P}$ by $Q T(\mathscr{P})$, however, we have the quasi-factorization property: for every invertible operator $A$ there exists a unitary operator $U$ such that $A=U S$ for some $S \in(Q T(\mathcal{P})) \cap(Q T(\mathscr{P}))^{-1}$.

COROLLARY 1.4. Every nest has the quasi-factorization property.

Proof. Let $\mathcal{P}$ be a nest, $A$ be an invertible operator, $Q_{P}$ be the projection onto $A P \mathcal{H}$, and $\mathcal{Z}=\left\{Q_{P}: P \in \mathscr{P}\right\}$. Let $\theta$ be the order isomorphism $P \rightarrow Q_{P}$. $\theta$ preserves dimensions, so by Theorem $1.3 \theta$ is implemented by an invertible operator $T=U+K$, with $U$ unitary and $K$ compact. Then $T^{-1}=U^{*}+L$ with $L$ compact. Now $T^{-1} A \in(\operatorname{alg} \mathscr{P}) \cap$ $(\text { alg } \mathcal{P})^{-1}$ since $T P \mathcal{H}=A P \mathcal{H}$ for all $P \in \mathcal{P}$, so $U^{*} A+L A, A^{-1} U+$ $A^{-1} K \in$ alg $\mathcal{P}$. Thus, $U^{*} A, A^{-1} U \in \operatorname{alg} \mathcal{P}+\mathscr{K}=Q T(\mathcal{P})$. Now let $S=$ $U^{*} A$. 
Another important result of Davidson (Theorem 1.5 below) which we will need gives necessary and sufficient conditions for the equality of two quasitriangular algebras. If $\mathscr{P}$ is a nest and $\tilde{P}$ is a finite rank projection in $\mathscr{\rho}^{\prime}$ (the commutant of $\mathscr{P}$ ), let $\mathcal{P}^{\tilde{P}}=\{0, P \vee \tilde{P}: P \in \mathscr{P}\} . \mathscr{P}^{\tilde{P}}$ is a nest, and is said to be a finite perturbation of $\mathscr{P}$. Note that $Q T(\mathscr{P})=Q T\left(\mathscr{P}^{\tilde{P}}\right)$, since if $T$ is in either alg $\mathcal{P}$ or alg $\mathcal{P} \tilde{P}$, then $\tilde{P}^{\perp} T \tilde{P}^{\perp}$ belongs to both.

Theorem 1.5. (Davidson, [8, Theorem 2.2]). For two nests 9 and 2 , the following are equivalent:

(1) $Q T(\mathscr{P})=Q T(\mathcal{Q})$

(2) There are finite perturbations $\mathscr{P}^{\tilde{P}}$ and $\mathcal{Q}^{\tilde{Q}}$ and a dimension-preserving order isomorphism $\theta: \mathcal{P} \tilde{P}^{\tilde{P}} \rightarrow \mathcal{Q}^{\tilde{Q}}$ such that $\theta-i d: \mathcal{P} \tilde{P} \rightarrow \mathcal{K}$ is strong-norm continuous.

(3) There are finite perturbations $\mathcal{P P}^{\tilde{P}}$ and $\mathscr{Q}^{\tilde{Q}}$ which are similar by $a$ compact perturbation of the identity.

(4) There are finite perturbations $\mathcal{P}^{\tilde{P}}$ and $\mathcal{Q}^{\tilde{Q}}$, an order isomorphism $\theta$ : $\mathscr{P}^{\tilde{P}} \rightarrow \mathcal{Q}^{\mathcal{Q}}$, and a sequence of unitary operators $U_{n}$ with $U_{n}-I \in \mathcal{K}$ such that $\theta=\left.\lim _{n \rightarrow \infty} \operatorname{Ad} U_{n}\right|_{\mathscr{P}} \tilde{P}$ uniformly in the norm on $\mathscr{P} \tilde{P}$.

REMARKS. The equivalence between (1) and (4) is essentially due to Andersen [1]. This equivalence is interesting because it yields the fact that two quasitriangular algebras are unitarily equivalent if and only if, after finite perturbations, the determining nests are "approximately" unitarily equivalent. The equivalence between (1) and (2) seems to be much more useful, however, since it allows one to make use of the characterization of quasitriangularity given in Theorem 1.2. Finally, the equivalence between (1) and (3), along with Theorem 1.3, gives the important connection between similarity of nests and unitary equivalence of quasitriangular algebras. Stated explicitly, two quasitriangular algebras are unitarily equivalent if and only if, after finite perturbations, the determining nests are similar. The similarity can be taken to be an arbitrarily small perturbation of a unitary, so again the nests are "almost" unitarily equivalent.

Finally, we will need several additional lemmas. If $\mathscr{P}$ and $\mathcal{L}$ are linearly ordered sets of projections (not necessarily nests), we say that $\mathscr{P}$ and $\mathcal{L}$ are compactly equivalent, denoted $\mathscr{P} \sim_{\mathcal{K}} \mathcal{Q}$, if the following two properties hold:

(i) For each $P \in \mathscr{P}$ there is some $Q \in \mathcal{Q}$ such that $P-Q \in \mathcal{K}$.

(ii) For each $Q^{\prime} \in \mathcal{Q}$ there is some $P^{\prime} \in \mathscr{P}$ such that $P^{\prime}-Q^{\prime} \in \mathscr{K}$. 
LeMma 1.6. Suppose $\mathscr{P} \sim_{\mathscr{K}} 2, P, P^{\prime} \in \mathcal{P}, Q, Q^{\prime} \in 2, P<P^{\prime}$, $\operatorname{dim}\left(P^{\prime}-P\right) \mathcal{H}=\infty$, and $P-Q, P^{\prime}-Q^{\prime} \in \mathcal{K}$. Then $Q<Q^{\prime}$ and $\operatorname{dim}\left(Q^{\prime}-Q\right) \mathcal{H}=\infty$.

Proof. $Q \neq Q^{\prime}$, because otherwise we would have $P^{\prime}-P=$ $\left(P^{\prime}-Q^{\prime}\right)+(Q-P) \in \mathcal{K}$, a contradiction. Now suppose $Q>Q^{\prime}$. Then $\left(P^{\prime}-P\right)+\left(Q-Q^{\prime}\right)=\left(P^{\prime}-Q^{\prime}\right)+(Q-P) \in \mathcal{K}$

$\Rightarrow \quad\left(P^{\prime}-P\right)=-\left(Q-Q^{\prime}\right)+K$ for some $K \in \mathcal{K}$

$\Rightarrow \pi\left(P^{\prime}-P\right)=\pi\left(-\left(Q-Q^{\prime}\right)\right), \quad$ where $\pi: \mathcal{L}(\mathcal{H}) \rightarrow \mathcal{L}(\mathcal{H}) / \mathcal{K}(\mathcal{H})$

is the canonical homomorphism. Therefore, $\sigma_{e}\left(P^{\prime}-P\right)=\sigma_{e}\left(-\left(Q-Q^{\prime}\right)\right)$, where $\sigma_{e}(T)$ denotes the essential spectrum of $T . P^{\prime}-P$ and $Q-Q^{\prime}$ are self-adjoint projections, so $\pi\left(P^{\prime}-P\right)$ and $\pi\left(Q-Q^{\prime}\right)$ are also. Thus, $\sigma_{e}\left(P^{\prime}-P\right) \subseteq\{0,1\}, \sigma_{e}\left(Q-Q^{\prime}\right) \subseteq\{0,1\}$, and

$$
\sigma_{e}\left(P^{\prime}-P\right)=\{0\} \Leftrightarrow \pi\left(P^{\prime}-P\right)=0 \Leftrightarrow P^{\prime}-P \in \mathscr{K} .
$$

Since $P^{\prime}-P \notin \mathscr{K}$, we have that

$$
\{1\} \subseteq \sigma_{e}\left(P^{\prime}-P\right)=\sigma_{e}\left(-\left(Q-Q^{\prime}\right)\right)=-\sigma_{e}\left(Q-Q^{\prime}\right) \subseteq\{0,-1\},
$$

which is a contradiction. Therefore, $Q<Q^{\prime}$. If $\operatorname{dim}\left(Q^{\prime}-Q\right) \mathcal{H}<\infty$, then $P^{\prime}-P=\left(P^{\prime}-Q^{\prime}\right)+\left(Q^{\prime}-Q\right)+(Q-P) \in \mathcal{K}$, a contradiction. Thus, $\operatorname{dim}\left(Q^{\prime}-Q\right) \mathcal{H}=\infty$.

LEMMA 1.7. Let $\left\{P_{1}, P_{2}, \ldots, P_{n}\right\} \sim \sim_{\kappa}\left\{Q_{1}, Q_{2}, \ldots, Q_{n}\right\}$ with $P_{1}<P_{2}<$ $\cdots<P_{n}$ and $Q_{1}<Q_{2}<\cdots<Q_{n}$. Suppose also that $\operatorname{dim}\left(P_{l}-P_{i-1}\right) \mathcal{H}=$ $\infty \Leftrightarrow \operatorname{dim}\left(Q_{l}-Q_{i-1}\right) \mathcal{H}=\infty$ for $2 \leq i \leq n$. Then $P_{i}-Q_{i} \in \mathcal{K}$ for all $i$, $1 \leq i \leq n$.

Proof. Suppose that $P_{1}-Q_{1} \notin \mathscr{K}$. Then $P_{1}-Q_{k} \in \mathscr{K}$ with $\operatorname{dim}\left(Q_{k}-Q_{1}\right) \mathcal{H}=\infty$ for some $k, 2 \leq k \leq n$, and $P_{J}-Q_{1} \in \mathcal{K}$ with $\operatorname{dim}\left(P_{j}-P_{1}\right) \mathcal{H}=\infty$ for some $j, 2 \leq j \leq n$. But this contradicts Lemma 1.6. Therefore, $P_{1}-Q_{1} \in \mathcal{K}$.

Now if $\operatorname{dim}\left(P_{2}-P_{1}\right) \mathcal{H}<\infty$ and $\operatorname{dim}\left(Q_{2}-Q_{1}\right) \mathcal{H}<\infty$, then clearly $P_{2}-Q_{2} \in \mathcal{K}$. Suppose $\operatorname{dim}\left(P_{2}-P_{1}\right) \mathcal{H}=\operatorname{dim}\left(Q_{2}-Q_{1}\right) \mathcal{H}=\infty$. Then $P_{1}-Q_{k} \notin \mathcal{K}$ and $P_{k}-Q_{1} \notin \mathscr{K}$ for all $k, 2 \leq k \leq n$. It follows that $\left\{P_{2}, \ldots, P_{n}\right\} \sim_{\mathcal{K}}\left\{Q_{2}, \ldots, Q_{n}\right\}$, and therefore, by the same reasoning used in the first paragraph above, $P_{2}-Q_{2} \in \mathscr{K}$. In a similar manner, it follows that $P_{3}-Q_{3} \in \mathcal{K}$, and then that $P_{4}-Q_{4} \in \mathcal{K}$, etc.

LEMMA 1.8. Let $\left\{E_{n}: 1 \leq n<\infty\right\}$ be a sequence of projections such that $E_{n} \rightarrow 0$ strongly, and let $K$ be any compact operator. Then $\left\|K E_{n}\right\| \rightarrow 0$ and $\left\|E_{n} K\right\| \rightarrow 0$. 
Proof. This lemma is well-known. First, suppose that $K$ is a rank one operator, so $K x=(x, e) f$ for some $e, f \in \mathcal{H}$. Then $\left\|E_{n} K\right\|=$ $\left\|(\cdot, e) E_{n} f\right\| \leq\|e\|\left\|E_{n} f\right\| \rightarrow 0$ and $\left\|K E_{n}\right\|=\left\|\left(\cdot, E_{n} e\right) f\right\| \leq\left\|E_{n} e\right\|\|f\|$ $\rightarrow 0$. For general $K$, approximate $K$ with finite linear combinations of rank one operators.

2. The derivation theorem. Our main result is Theorem 2.1 below, from which the derivation theorem (2.2) follows.

THEOREM 2.1. Let $\mathcal{P}$ be a nest, and let $\alpha$ be an automorphism of $Q T(\mathcal{P})$ such that $\|\alpha-i d\|<\frac{1}{2}$. Then $\alpha$ is inner.

(Note: This implies that the quotient topology on $\operatorname{Out}(Q T(\mathcal{P}))=$ automorphisms/inner automorphisms is discrete.)

We will give the proof below. First, however, we will obtain the derivation theorem.

THEOREM 2.2. Let $\mathcal{P}$ be a nest. Then every derivation $\delta: Q T(\mathscr{P}) \rightarrow$ $Q T(\mathcal{P})$ is inner.

Proof. Note: The proof is a slight variation of the argument given by Christensen and Peligrad [4] for the special case in which $\mathscr{P}$ consists of an increasing sequence $\left\{P_{n}: 1 \leq n<\infty\right\}$ of finite rank projections such that $P_{n} \rightarrow I$ strongly.

Let $\delta: Q T(\mathcal{P}) \rightarrow Q T(\mathcal{P})$ be a derivation. Then $\left.\delta\right|_{\mathscr{K}}$ is continuous by [13, Lemma 1.2]. Moreover $\delta(K) \in \mathscr{K}$ for $K \in \mathcal{K}$. To see this, let $\left\{P_{n}\right.$ : $1 \leq n<\infty\}$ be any increasing sequence of finite rank projections such that $P_{n} \rightarrow I$ strongly. Then $\left\|K-K P_{n}\right\| \rightarrow 0$ as $n \rightarrow \infty$ by Lemma 1.8. Therefore,

$$
\left\|\delta(K)-\left(\delta(K) P_{n}+K \delta\left(P_{n}\right)\right)\right\|=\left\|\delta(K)-\delta\left(K P_{n}\right)\right\|=\left\|\delta\left(K-K P_{n}\right)\right\| \rightarrow 0
$$

since $\left.\delta\right|_{\mathscr{K}}$ is continuous. Since $\delta(K) P_{n}+K \delta\left(P_{n}\right) \in \mathscr{K}$ for each $n$, it follows that $\delta(K) \in \mathscr{K}$. Therefore, $\left.\delta\right|_{\mathscr{K}}: \mathscr{K} \rightarrow \mathscr{K}$ is a continuous derivation, so $\left.\delta\right|_{\mathcal{H}}=\left.(\operatorname{ad} X)\right|_{\mathcal{K}}$ for some $X \in \mathcal{L}(\mathcal{H})$ by [14, Theorem 4]. Now if $T \in Q T(\mathscr{P})$ and $K \in \mathcal{K}$, then

$$
\begin{aligned}
\delta(T) K+ & T \delta(K)=\delta(T K)=X T K-T K X \\
& =(X T-T X) K+T(X K-K X) \\
& =(X T-T X) K+T \delta(K) \Rightarrow \delta(T) K=(X T-T X) K .
\end{aligned}
$$

Let $K=P_{n}$ and take strong limits as $n \rightarrow \infty$. It follows that $\delta(T)=$ $X T-T X$, so $\delta=\operatorname{ad} X$. 
It remains to show that $X \in Q T(\mathcal{P}) . \delta$ generates the uniformly continuous automorphism group $\{\exp (t \delta): t \in \mathbf{R}\}$ on $Q T(\mathscr{P})$, where $\exp (t \delta)(T)=(\exp (t X)) T(\exp (-t X))$. Thus, there is some $t_{0}$ such that $\|\exp (t \delta)-i d\|<\frac{1}{2}$ for $|t|<t_{0}$, so by Theorem $2.1, \exp (t \delta)$ is inner for all $|t|<t_{0}$. Therefore, if $|t|<t_{0}$, then $(\exp (t X)) T(\exp (-t X))=A T A^{-1}$ for some operator $A$ such that $A, A^{-1} \in Q T(\mathcal{P})$, and for all $T \in Q T(\mathcal{P})$. Then $A^{-1}(\exp (t X)) T=T A^{-1}(\exp (t X))$ for all $T \in Q T(\mathscr{P})$, so $A^{-1}(\exp (t X)) \in(Q T(\mathscr{P}))^{\prime}=\lambda \mathbf{C}($ since $Q T(\mathscr{P}) \supseteq \mathscr{K})$. Thus, $\exp (t X)=$ $\lambda A$ for some $\lambda \in \mathbf{C}$, so $\exp (t X) \in Q T(\mathscr{P})$ for all $|t|<t_{0}$. Now, by taking the derivative of the function $t \rightarrow \exp (t X)$ at $t=0$, it follows that $X \in$ $Q T(\mathcal{P})$.

Proof of Theorem 2.1. The proof is long, so it will be divided into several steps.

Step 1. Since $Q T(\mathscr{P}) \supseteq$ finite rank operators\}, it follows by [17, Theorem 2.5.19] that $\alpha=\operatorname{Ad} A$ for some invertible operator $A \in \mathcal{L}(\mathcal{H})$. By Corollary 1.4, there is a unitary operator $U$ such that $U^{*} A, A^{-1} U \in$ $Q T(\mathcal{P})$. Therefore, $\operatorname{Ad}\left(A^{-1} U\right)$ is an inner automorphism of $Q T(\mathscr{P})$, so $\operatorname{Ad} U$ is an automorphism of $Q T(\mathscr{P})$ since $\operatorname{Ad} U=(\operatorname{Ad} A) \circ\left(\operatorname{Ad}\left(A^{-1} U\right)\right)$. Also, $\alpha$ is inner if and only if $\operatorname{Ad} U$ is inner. The remainder of the proof consists of showing that $U, U^{*} \in Q T(\mathscr{P})$, thus proving that $\alpha$ is inner.

Let $Q_{P}=U P U^{*}$ and let $\mathcal{L}=\left\{Q_{P}: P \in \mathscr{P}\right\}$. Then 2 is a nest and $Q T(\mathscr{P})=(\operatorname{Ad} U)(Q T(\mathscr{P}))=U(Q T(\mathscr{P})) U^{*}=Q T\left(U \mathscr{P} U^{*}\right)=Q T(\mathscr{Q})$. Therefore, by Theorem 1.5, there are finite rank projections $\tilde{P} \in \mathscr{P}^{\prime}$ and $\tilde{Q} \in \mathcal{Q}^{\prime}$ and a dimension-preserving order isomorphism $\theta: \rho^{\tilde{P}} \rightarrow \mathscr{Q}^{\tilde{Q}}$ such that $\theta-i d: \mathscr{P}^{\tilde{P}} \rightarrow \mathscr{K}$ is strong-norm continuous.

Step 2. Suppose that $P_{0}$ is a left limit point of $\mathscr{P}$, so there is a sequence $\left\{P_{n}: 1 \leq n<\infty\right\} \subseteq \mathcal{P}$ such that $P_{n}<P_{n+1}<P_{0}$ for all $n$ and $P_{n} \rightarrow P_{0}$ strongly. In this step we will show that there is some $N>0$ such that $n \geq N \Rightarrow \theta\left(P_{n} \vee \tilde{P}\right)=Q_{P_{n}} \vee \tilde{Q}=U P_{n} U^{*} \vee \tilde{Q}$. By the continuity of $\theta$, we then also have that $\theta\left(P_{0} \vee \tilde{P}\right)=U P_{0} U^{*} \vee \tilde{Q}$. Also, the same result is true if $P_{0}$ is a right limit point and $\left\{P_{n}: 1 \leq n<\infty\right\} \subseteq \mathcal{P}$ is a decreasing sequence with $P_{n} \rightarrow P_{0}$ strongly, and the proof is similar.

By Lemma $1.8,\left\|\left(P_{0}-P_{n}\right) \tilde{P}\right\| \rightarrow 0$ as $n \rightarrow \infty$, so $\left\|\left(P_{0}-P_{n}\right) \tilde{P}\right\|=0$ for $n \geq$ some $N_{1}$ since $\left(P_{0}-P_{n}\right) \tilde{P}$ is a projection. Thus, $P_{0} \tilde{P}=P_{n} \tilde{P}$ for $n \geq N_{1}$, so

$$
P_{n} \vee \tilde{P}<P_{n+1} \vee \tilde{P}<P_{0} \vee \tilde{P} \quad \text { for } n \geq N_{1}
$$


(note that $P_{n} \vee \tilde{P}=P_{n}+\tilde{P}-P_{n} \tilde{P}$ ). Similarly, $U P_{0} U^{*} \tilde{Q}=U P_{n} U^{*} \tilde{Q}$ for $n \geq N_{2}$, some $N_{2} \geq N_{1}$, so

$$
U P_{n} U^{*} \vee \tilde{Q}<U P_{n+1} U^{*} \vee \tilde{Q}<U P_{0} U^{*} \vee \tilde{Q} \quad \text { for } n \geq N_{2} .
$$

Also, $P_{n} \vee \tilde{P} \rightarrow P_{0} \vee \tilde{P}$ and $U P_{n} U^{*} \vee \tilde{Q} \rightarrow U P_{0} U^{*} \vee \tilde{Q}$ strongly.

Let $E_{n}=\left(P_{0} \vee \tilde{P}\right)-\left(P_{n} \vee \tilde{P}\right), F_{n}=\left(U P_{0} U^{*} \vee \tilde{Q}\right)-\left(U P_{n} U^{*} \vee \tilde{Q}\right)$, and $E_{n}^{\prime}=\theta^{-1}\left(U P_{0} U^{*} \vee \tilde{Q}\right)_{\tilde{P}}-\theta^{-1}\left(U P_{n} U^{*} \vee \tilde{Q}\right)$, for $n \geq N_{2}$. Let $r=$ $\|\alpha-i d\|$. Since $E_{n}^{\prime} \in Q T\left(\mathcal{P}^{\tilde{P}}\right)=Q T(\mathscr{P})$, we have

$$
\left\|A E_{n}^{\prime} A^{-1}-E_{n}^{\prime}\right\| \leq r<\frac{1}{2} \text { for all } n \geq N_{2} \text {. }
$$

Since $\theta-i d$ is strong-norm continuous, it follows that there is some $N_{3} \geq N_{2}$ such that $n \geq N_{3} \Rightarrow\left\|E_{n}^{\prime}-F_{n}\right\| \leq \frac{1}{2}\left(\frac{1}{2}-r\right)$. Therefore, $n \geq N_{3} \Rightarrow$ $\left\|A E_{n}^{\prime} A^{-1}-F_{n}\right\| \leq r+\frac{1}{2}\left(\frac{1}{2}-r\right)$, and thus

$$
\left\|U^{*} A E_{n}^{\prime} A^{-1} U-U^{*} F_{n} U\right\| \leq r+\frac{1}{2}\left(\frac{1}{2}-r\right) .
$$

But $U^{*} F_{n} U=E_{n}$ for $n \geq N_{3}$. To see this, note that since $P_{0} \tilde{P}=P_{n} \tilde{P}$, we have

$$
P_{0}-P_{n}=P_{0} \tilde{P}^{\perp}-P_{n} \tilde{P}^{\perp}=\left(P_{0} \tilde{P}^{\perp}+\tilde{P}\right)-\left(P_{n} \tilde{P}^{\perp}+\tilde{P}\right)=E_{n}
$$

Similarly, since $U P_{0} U^{*} \tilde{Q}=U P_{n} U^{*} \tilde{Q}$, we have $U P_{0} U^{*}-U P_{n} U^{*}=F_{n}$. Therefore, $U E_{n} U^{*}=U\left(P_{0}-P_{n}\right) U^{*}=F_{n}$. It follows that

$$
\left\|U^{*} A E_{n}^{\prime} A^{-1} U-E_{n}\right\| \leq r+\frac{1}{2}\left(\frac{1}{2}-r\right), \text { for } n \geq N_{3} .
$$

Now $U^{*} A, A^{-1} U \in Q T(\mathscr{P})=Q T\left(\mathscr{P}^{\tilde{P}}\right)$, so there are operators $X, Y$ $\in \operatorname{alg} \mathscr{P}^{\tilde{P}}$ and compact operators $K_{1}$ and $K_{2}$ such that $U^{*} A=X+K_{1}$ and $A^{-1} U=Y+K_{2}$. It follows by Lemma 1.8 that $\left\|K_{1} E_{n}^{\prime}\right\|,\left\|E_{n}^{\prime} K_{2}\right\| \rightarrow 0$, so there is some $N_{4} \geq N_{3}$ such that

$$
n \geq N_{4} \Rightarrow\left\|K_{1} E_{n}^{\prime}\right\|\|Y\|+\left\|U^{*} A\right\|\left\|E_{n}^{\prime} K_{2}\right\|<\frac{1}{2}\left(\frac{1}{2}-r\right) .
$$

Therefore,

$$
\begin{aligned}
n & \geq N_{4} \Rightarrow\left\|X E_{n}^{\prime} Y-E_{n}\right\| \\
& \leq\left\|U^{*} A E_{n}^{\prime} A^{-1} U-E_{n}\right\|+\left\|K_{1} E_{n}^{\prime}\right\|\|Y\|+\left\|U^{*} A\right\|\left\|E_{n}^{\prime} K_{2}\right\| \\
& <r+\frac{1}{2}\left(\frac{1}{2}-r\right)+\frac{1}{2}\left(\frac{1}{2}-r\right)=\frac{1}{2} .
\end{aligned}
$$


We then have that

$$
n \geq m \geq N_{4} \Rightarrow\left\|X E_{n-m}^{\prime} Y-E_{n-m}\right\|<1,
$$

where $E_{n-m}=E_{m}-E_{n}=\left(P_{n} \vee \tilde{P}\right)-\left(P_{m} \vee \tilde{P}\right)$, and $E_{n-m}^{\prime}=E_{m}^{\prime}-E_{n}^{\prime}$ $=\boldsymbol{\theta}^{-1}\left(U P_{n} U^{*} \vee \tilde{Q}\right)-\boldsymbol{\theta}^{-1}\left(U P_{m} U^{*} \vee \tilde{Q}\right)$.

If we can show that $E_{n-m}=E_{n-m}^{\prime}$ for all $n>m>N_{4}$, then it follows that $\theta\left(P_{n} \vee \tilde{P}\right)=U P_{n} U^{*} \vee \tilde{Q}$ for all $n \geq N=N_{4}+1$, which is what we set out to prove in this step. First, suppose that there is some $n>m \geq N_{4}$ such that the lower endpoint $R$ of $E_{n-m}$ is strictly smaller than the lower endpoint $R^{\prime}$ of $E_{n-m}^{\prime}$. Thus, there is some $\zeta \in E_{n-m} \mathcal{H},\|\zeta\|=1$, such that $R^{\prime \perp} \zeta=0$. Then $Y \zeta \in R^{\prime} \mathcal{H}$, so $X E_{n-m}^{\prime} Y \zeta=0$ and $\left\|\left(X E_{n-m}^{\prime} Y-E_{n-m}\right) \zeta\right\|$ $=1$, a contradiction. Now suppose there is some $n>m \geq N_{4}$ such that the upper endpoint $S$ of $E_{n-m}$ is strictly greater than the upper endpoint $S^{\prime}$ of $E_{n-m}^{\prime}$. Then there is some $\xi \in E_{n-m} \mathcal{H},\|\xi\|=1$, such that $S^{\prime} \xi=0$. Let $\lambda=X E_{n-m}^{\prime} Y \xi . \lambda \in S^{\prime} \mathcal{H}$, so $\lambda \perp \xi$ and

$$
1 \leq\|\lambda-\xi\|=\left\|\left(X E_{n-m}^{\prime} Y-E_{n-m}\right) \xi\right\|,
$$

a contradiction. Since the upper endpoint of $E_{n-m}$ is the lower endpoint of $E_{q-n}$, it follows that $E_{n-m}=E_{n-m}^{\prime}$ for all $n>m>N_{4}$.

Step 3. We are now ready to show that $U, U^{*} \in Q T(\mathscr{P})$. First, we will show in this step that $P \in \mathscr{P} \rightarrow P^{\perp} U P$ and $P \in \mathscr{P} \rightarrow P^{\perp} U^{*} P$ are strongnorm continuous functions.

$\theta-i d: \mathscr{P}^{\tilde{P}} \rightarrow \mathscr{K}$ is strong-norm continuous by Step 1, so it follows that $P \in \mathscr{P} \rightarrow \theta(P \vee \tilde{P})-P \vee \tilde{P} \in \mathcal{K}$ is strong-norm continuous (since $P \rightarrow P \vee \tilde{P}$ is strongly continuous). Now suppose $P_{n} \rightarrow P$ strongly. Then

$$
\begin{aligned}
\|\left(P^{\perp} \theta(\right. & \left.P \vee \tilde{P})-P^{\perp}(P \vee \tilde{P})\right)-\left(P_{n}^{\perp} \theta\left(P_{n} \vee \tilde{P}\right)-P_{n}^{\perp}\left(P_{n} \vee \tilde{P}\right)\right) \| \\
\leq & \left\|\left(P^{\perp}-P_{n}^{\perp}\right)(\theta(P \vee \tilde{P})-P \vee \tilde{P})\right\| \\
& \quad+\left\|P_{n}^{\perp}\right\|\left\|(\theta(P \vee \tilde{P})-P \vee \tilde{P})-\left(\theta\left(P_{n} \vee \tilde{P}\right)-P_{n} \vee \tilde{P}\right)\right\| \\
& \rightarrow 0 \quad \text { as } n \rightarrow \infty
\end{aligned}
$$

by Lemma 1.8 since $\theta(P \vee \tilde{P})-P \vee \tilde{P} \in \mathscr{K}$. Therefore, the map $P \in \mathcal{P}$ $\rightarrow P^{\perp} \theta(P \vee \tilde{P})-P^{\perp}(P \vee \tilde{P}) \in \mathscr{K}$ is strong-norm continuous (note that sequential continuity is sufficient since $\mathscr{P}$ is metrizable). It follows that $P \in \mathscr{P} \rightarrow P^{\perp} \theta(P \vee \tilde{P}) \in \mathscr{K}$ is strong-norm continuous, since $P \in \mathscr{P} \rightarrow$ $P^{\perp}(P \vee \tilde{P})=P^{\perp} \tilde{P} \in \mathcal{K}$ is strong-norm continuous by Lemma 1.8.

Now suppose $P_{0}$ is a left limit point of $\mathscr{P}$, and $\left\{P_{n}: 1 \leq n<\infty\right\} \subseteq \mathscr{P}$ is an increasing sequence which converges strongly to $P_{0}$. We know 
by Step 2 that there is some $N>0$ such that $n \geq N \Rightarrow \theta\left(P_{n} \vee \tilde{P}\right)=$ $U P_{n} U^{*} \vee \tilde{Q}$, and also that $\theta\left(P_{0} \vee \tilde{P}\right)=U P_{0} U^{*} \vee \tilde{Q}$. Then

$$
\begin{aligned}
n \geq & N \Rightarrow\left\|P_{0}^{\perp} U P_{0}-P_{n}^{\perp} U P_{n}\right\|=\left\|\left(P_{0}^{\perp} U P_{0}-P_{n}^{\perp} U P_{n}\right) U^{*}\right\| \\
\leq & \left\|\left(P_{0}^{\perp} U P_{0}-P_{n}^{\perp} U P_{n}\right) U^{*}+\left(P_{0}^{\perp} U P_{0}^{\perp} U^{*}-P_{n}^{\perp} U P_{n}^{\perp} U^{*}\right) \tilde{Q}\right\| \\
& +\left\|\left(P_{0}^{\perp} U P_{0}^{\perp} U^{*}-P_{n}^{\perp} U P_{n}^{\perp} U^{*}\right) \tilde{Q}\right\| \\
= & \left\|P_{0}^{\perp}\left(U P_{0} U^{*} \vee \tilde{Q}\right)-P_{n}^{\perp}\left(U P_{n} U^{*} \vee \tilde{Q}\right)\right\| \\
& +\left\|\left(P_{0}^{\perp} U P_{0}^{\perp} U^{*}-P_{n}^{\perp} U P_{n}^{\perp} U^{*}\right) \tilde{Q}\right\| \\
= & \left\|P_{0}^{\perp} \theta\left(P_{0} \vee \tilde{P}\right)-P_{n}^{\perp} \theta\left(P_{n} \vee \tilde{P}\right)\right\|+\left\|\left(P_{0}^{\perp} U P_{0}^{\perp} U^{*}-P_{n}^{\perp} U P_{n}^{\perp} U^{*}\right) \tilde{Q}\right\| .
\end{aligned}
$$

The first term converges to 0 as $n \rightarrow \infty$ since $P \rightarrow P^{\perp} \theta(P \vee \tilde{P})$ is strongnorm continuous, and the second term converges to 0 by Lemma 1.8. Therefore, $P_{n}^{\perp} U P_{n} \rightarrow P_{0}^{\perp} U P_{0}$ as $n \rightarrow \infty$.

If $P_{0}^{\prime}$ is a right limit point of $\mathscr{P}$ and $\left\{P_{n}^{\prime}: 1 \leq n<\infty\right\} \subseteq \mathscr{P}$ is a decreasing sequence which converges strongly to $P_{0}^{\prime}$, then a similar argument shows that $P_{n}^{\prime \perp} U P_{n}^{\prime} \rightarrow P_{0}^{\prime \perp} U P_{0}^{\prime}$ as $n \rightarrow \infty$. Therefore, the map $P \in \mathscr{P} \rightarrow P^{\perp} U P$ is strong-norm continuous.

To show that $P \in \mathscr{P} \rightarrow P^{\perp} U^{*} P$ is strong-norm continuous, first note that $P \in \mathscr{P} \rightarrow U^{*} \theta(P \vee \tilde{P})-U^{*}(P \vee \tilde{P}) \in \mathscr{K}$ is strong-norm continuous, and then that $P \in \mathscr{P} \rightarrow P^{\perp} U^{*} \theta(P \vee \tilde{P})-P^{\perp} U^{*}(P \vee \tilde{P}) \in \mathcal{K}$ and $P \in \mathcal{P} \rightarrow P^{\perp} U^{*} \theta(P \vee \tilde{P})-P^{\perp} U^{*} P \in \mathscr{K}$ are strong-norm continuous. An argument similar to the one given above (i.e., by considering left and right limit points) shows that $P \in \mathcal{P} \rightarrow P^{\perp} U^{*} \theta(P \vee \tilde{P})$ is strong-norm continuous, and the result follows.

Step 4. In this step we will show that $P^{\perp} U P \in \mathcal{K}$ and $P^{\perp} U^{*} P \in \mathscr{K}$ for all $P \in \mathscr{P}$. It will then follow, by Step 3 and Theorem 1.2, that $U, U^{*} \in Q T(\mathscr{P})$.

If $P_{0}$ is a limit point of $\mathscr{P}$, then $P_{0}^{\perp}\left(U P_{0} U^{*} \vee \tilde{Q}\right)=P_{0}^{\perp} \theta\left(P_{0} \vee \tilde{P}\right)$ by Step 2. Since

$$
P_{0}^{\perp} \theta\left(P_{0} \vee \tilde{P}\right)=P_{0}^{\perp}\left(\theta\left(P_{0} \vee \tilde{P}\right)-P_{0} \vee \tilde{P}\right)+P_{0}^{\perp}\left(P_{0} \vee \tilde{P}\right) \in \mathscr{K}
$$

and $\tilde{Q} \in \mathscr{K}$, it follows that $P_{0}^{\perp} U P_{0} \in \mathscr{K}$. Also,

$$
\begin{aligned}
P_{0}^{\perp} U^{*}\left(P_{0} \vee \tilde{P}\right)= & P_{0}^{\perp} U^{*} \theta\left(P_{0} \vee \tilde{P}\right) \\
& -\left(P_{0}^{\perp} U^{*}\left(\theta\left(P_{0} \vee \tilde{P}\right)-P \vee \tilde{P}\right)\right) \in \mathscr{K}
\end{aligned}
$$


since

$$
\begin{aligned}
P_{0}^{\perp} U^{*} \theta\left(P_{0} \vee \tilde{P}\right) & =P_{0}^{\perp} U^{*}\left(U P_{0} U^{*} \vee \tilde{Q}\right) \\
& =P_{0}^{\perp} U^{*}\left(U P_{0} U^{*}+U P_{0}^{\perp} U^{*} \tilde{Q}\right)=P_{0}^{\perp} U^{*} \tilde{Q}
\end{aligned}
$$

It follows that $P_{0}^{\perp} U^{*} P_{0} \in \mathscr{K}$ since $\tilde{P} \in \mathcal{K}$.

Now suppose $P_{0}$ is an isolated point of $\mathscr{P}$. Let $\mathscr{R}=\left\{P \in \mathscr{P}: P<P_{0}\right.$ and $P$ is a limit point of $\mathscr{P}\}$ and let $\mathcal{S}=\left\{P \in \mathcal{P}: P>P_{0}\right.$ and $P$ is a limit point of $\mathcal{P}\}$. If $\Re \neq \varnothing$, let $R^{\prime}=\bigvee\{P: P \in \mathcal{R}\}$. Then $R^{\prime}$ is a limit point of $\mathscr{P}$. If $R^{\prime}$ is a right limit point, then by Step 2 there is some $R^{\prime \prime} \in \mathscr{P}$, $R^{\prime}<R^{\prime \prime}<P_{0}$, such that $\theta\left(R^{\prime \prime} \vee \tilde{P}\right)=U R^{\prime \prime} U^{*} \vee \tilde{Q}$. Let $R=R^{\prime \prime}$. If $R^{\prime}$ is not a right limit point, let $R=R^{\prime}$. Finally, if $R=\varnothing$, let $R=0$. Then $\theta(R \vee \tilde{P})=U R U^{*} \vee \tilde{Q}$ and $\left\{P \in \mathscr{P}: R \leq P \leq P_{0}\right\}$ is finite. Similarly, if $\mathcal{\delta} \neq \varnothing$, let $S^{\prime}=\wedge\{P: P \in \mathcal{S}\}$. Then $S^{\prime}$ is a limit point of $\mathcal{P}$. If $S^{\prime}$ is a left limit point, then by Step 2 there is some $S^{\prime \prime} \in \mathcal{P}, P_{0}<S^{\prime \prime}<S^{\prime}$, such that $\theta\left(S^{\prime \prime} \vee \tilde{P}\right)=U S^{\prime \prime} U^{*} \vee \tilde{Q}$. Let $S=S^{\prime \prime}$. If $S^{\prime}$ is not a left limit point, let $S=S^{\prime}$. Finally, if $\delta=\varnothing$, let $S=I$. Then $\theta(S \vee \tilde{P})=U S U^{*} \vee \tilde{Q}$ and $\left\{P \in \mathcal{P}: P_{0} \leq P \leq S\right\}$ is finite. Therefore, $\{P \in \mathscr{P}: R \leq P \leq S\}$ is finite and thus has the form $R=R_{0}<R_{1}<R_{2}<\cdots<R_{n}=S$. Now $\theta\left(R_{i} \vee \tilde{P}\right)-R_{i} \vee \tilde{P} \in \mathscr{K}$ for all $i, 0 \leq i \leq n$, so

$$
\begin{aligned}
\left\{R_{i} \vee \tilde{P}: 0 \leq i \leq n\right\} & \sim_{\mathcal{K}}\left\{\theta\left(R_{i} \vee \tilde{P}\right): 0 \leq i \leq n\right\} \\
& =\left\{U R_{i} U^{*} \vee \tilde{Q}: 0 \leq i \leq n\right\}
\end{aligned}
$$

since $\theta(R \vee \tilde{P})=U R U^{*} \vee \tilde{Q}$ and $\theta(S \vee \tilde{P})=U S U^{*} \vee \tilde{Q}$. Therefore, $\left\{R_{i}: 0 \leq i \leq n\right\} \sim \sim_{K}\left\{U R_{i} U^{*}: 0 \leq i \leq n\right\}$, so by Lemma 1.7 we have $R_{i}-$ $U R_{l} U^{*} \in \mathscr{K}$ for all $i, 0 \leq i \leq n$, since $U$ is unitary. Thus, $P_{0}-U P_{0} U^{*} \in$ $\mathcal{K}$, since $P_{0}=R_{k}$ for some $k$, from which it follows that $P_{0}^{\perp} U P_{0} \in \mathcal{K}$ and $P_{0}^{\perp} U^{*} P_{0} \in \mathscr{K}$.

Step 5. By Step 3, the functions $P \in \mathcal{P} \rightarrow P^{\perp} U P$ and $P \in \mathscr{P} \rightarrow$ $P^{\perp} U^{*} P$ are strong-norm continuous. By Step 4, $P^{\perp} U P \in \mathcal{K}$ and $P^{\perp} U^{*} P$ $\in \mathcal{K}$ for all $P \in \mathscr{P}$. Therefore, $U, U^{*} \in Q T(\mathscr{P})$ by Theorem 1.2, and thus $\alpha$ is inner by Step 1 .

There are several immediate consequences of Theorem 2.2, including improvements (Corollaries 2.4 and 2.5 below) of Theorem 2.1.

COROllary 2.3. Let $\mathcal{P}$ be a nest, and let $\delta$ be a derivation of $Q T(\mathcal{P})$. Then the function $P \in \mathscr{P} \rightarrow P^{\perp} \delta(P) P \in \mathcal{K}$ is strong-norm continuous. 
Proof. By Theorem 2.2, $\delta=$ ad $X$ for some $X \in Q T(\mathcal{P})$. Therefore, $P \in \mathscr{P} \rightarrow P^{\perp} X P \in \mathscr{K}$ is strong-norm continuous by Theorem 1.2, and the result follows since $P^{\perp} \delta(P) P=P^{\perp}(X P-P X) P=P^{\perp} X P$.

COROLlaRy 2.4. Let $\mathcal{P}$ be a nest, and let $\alpha$ be an automorphism of $Q T(\mathcal{P})$ such that $\sigma(\alpha)$, the spectrum of $\alpha$, is contained in $\Omega=\{z \in \mathbf{C}$ : $\operatorname{Re} z>0\}$. Then $\alpha$ is inner.

Proof. By [17, Theorem 2.5.19], $\alpha=\operatorname{Ad} A$ for some invertible $A \in$ $\mathcal{L}(\mathcal{H})$, so $\alpha$ is continuous. Therefore, by [19], there is a derivation $\delta$ of $Q T(\mathcal{P})$ such that $\alpha=\exp (t \delta)$ for some $t \in \mathbf{R}$. By Theorem 2.2, $\delta$ is inner, so $\delta=$ ad $X$ for some $X \in Q T(\mathcal{P})$. Thus, $\exp (t X) \in Q T(\mathcal{P})$ for all $t \in \mathbf{R}$ and $\alpha=\operatorname{Ad}(\exp (t X))$, so $\alpha$ is inner.

COROLlaRY 2.5. Let $\rho$ be a nest, and let $\alpha$ be an automorphism of $Q T(\mathscr{P})$ such that $\|\alpha-i d\|<1$. Then $\alpha$ is inner.

Proof. The result follows from Corollary 2.4 since $\|\alpha-i d\|<1 \Rightarrow$ $\sigma(\alpha) \subseteq \Omega$.

Addendum (June, 1983). Since this paper was written, Kenneth R. Davidson and the author have prepared a joint paper, Automorphisms of Quasitriangular Algebras, which contains a different proof of Theorem 2.2. The new proof follows fairly quickly from a complete analysis of the outer automorphism groups of quasitriangular algebras.

\section{REFERENCES}

[1] N. T. Andersen, Compact perturbations of reflexive algebras, J. Functional Analysis, 38 (1980), 366-400.

[2] W. B. Arveson, Interpolation problems in nest algebras, J. Functional Analysis, 20 (1975), 208-233.

[3] E. Christensen, Derivations of nest algebras, Math. Ann., 229 (1977), 155-161.

[4] E. Christensen and C. Peligrad, Commutants of nest algebras modulo the compact operators, Københavns Universitet Matematisk Institut Preprint Series \#31, Nov. 1978.

[5] K. R. Davidson, Commutative subspace lattices, Indiana Univ. Math. J., 27 (1978), 479-490.

[6] Compact perturbations of reflexive algebras, Canad. J. Math., 33 (1981), 685-700.

[7] _ Quasitriangular algebras are "maximal", J. Operator Theory, (to appear).

[8] Similarity and compact perturbations of nest algebras, J. Reine Angew. Math., (to appear). 
[9] J. A. Erdos, Non-selfadjoint Operator Algebras, Proc. Royal Irish Academy, Sect. A, No. 1, 81 (1981), 127-145.

[10] __ Unitary invariants for nests, Pacific J. Math., 23 (1967), 229-256.

[11] T. Fall, W. Arveson and P. Muhly, Perturbations of nest algebras, J. Operator Theory, 1 (1979), 137-150.

[12] F. Gilfeather, A. Hopenwasser and D. Larson, Reflexive algebras with finite width lattices: tensor products, cohomology, compact perturbations, preprint.

[13] B. E. Johnson and S. K. Parrott, Operators commuting with a von Neumann algebra modulo the set of compact operators, J. Functional Analysis, 11 (1972), 39-61.

[14] R. V. Kadison, Derivations of operator algebras, Ann. Math., 83 (1966), 280-293.

[15] D. R. Larson, Nest algebras and similarity transformations, Ann. Math., (to appear).

[16] J. K. Plastiras, Quasitriangular operator algebras, Pacific J. Math., 64 (1976), 543-549.

[17] C. E. Rickart, General Theory of Banach Algebras, D. von Nostrand Company, Ltd. (1960).

[18] B. H. Wagner, Automorphisms and derivations of certain operator algebras, Doctoral dissertation, University of California, Berkeley (1982).

[19] G. Zeller-Meier, Sur les Automorphismes des Algebres de Banach, C. R. Acad. Sci. Paris, 264 (1967), 1131-1132.

Received March 28, 1983.

UNIVERSITY OF CALIFORNIA

DAVIS, CA 95616 



\section{PACIFIC JOURNAL OF MATHEMATICS \\ EDITORS}

DONALD BABBITt (Managing Editor)

University of California

Los Angeles, CA 90024

J. Dugundu

University of Southern California

Los Angeles, CA 90089-1113

R. FINN

Stanford University

Stanford, CA 94305

HERMANN FLASCHKA

University of Arizona

Tucson, AZ 85721
C. C. MOORE

University of California

Berkeley, CA 94720

ARTHur Ogus

University of California

Berkeley, CA 94720

Hugo RossI

University of Utah

Salt Lake City, UT 84112

H. SAMELSON

Stanford University

Stanford, CA 94305

ASSOCIATE EDITORS

R. ARENS

E. F. BECKENBACH

B. H. NeumanN (1906-1982)

F. WOLF

K. YoshidA

\section{SUPPORTING INSTITUTIONS}

UNIVERSITY OF ARIZONA

UNIVERSITY OF BRITISH COLUMBIA

UNIVERSITY OF OREGON

CALIFORNIA INSTITUTE OF TECHNOLOGY

UNIVERSITY OF CALIFORNIA

MONTANA STATE UNIVERSITY

UNIVERSITY OF SOUTHERN CALIFORNIA

UNIVERSITY OF NEVADA, RENO

STANFORD UNIVERSITY

UNIVERSITY OF HAWAII

NEW MEXICO STATE UNIVERSITY

UNIVERSITY OF TOKYO

UNIVERSITY OF UTAH

OREGON STATE UNIVERSITY

WASHINGTON STATE UNIVERSITY

UNIVERSITY OF WASHINGTON 


\section{Pacific Journal of Mathematics}

Vol. 114, No. $1 \quad$ May, 1984

David Marion Arnold and Charles Irvin Vinsonhaler, Typesets and cotypesets of rank-2 torsion free abelian groups $\ldots \ldots \ldots \ldots \ldots \ldots \ldots 1$

Duncan Alan Buell and Richard Howard Hudson, Solutions of certain

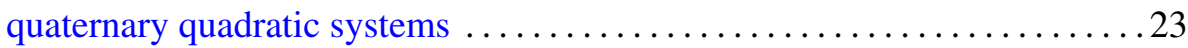

Hans Delfs and Manfred Knebusch, Separation, retractions and homotopy

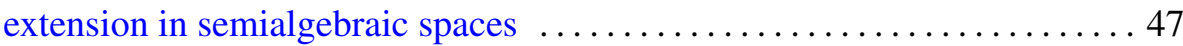

I. Erdélyi and Sheng-Wang Wang, A spectral duality theorem for closed

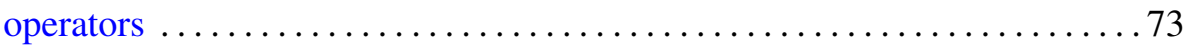

Theodore William Gamelin, Weak compactness of representing measures for $R(K)$

Kenneth R. Goodearl and T. H. Lenagan, Krull dimension of

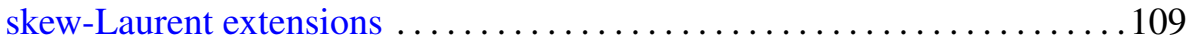

Daniel Joseph Gross, Compact quotients by $\mathbf{C}^{*}$-actions . . . . . . . . . . . 149

Goo Ishikawa, Satoshi Koike and Masahiro Shiota, Critical value sets of

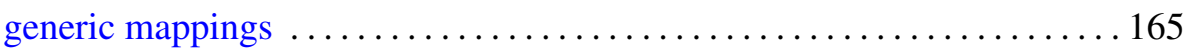

Hong Oh Kim, Derivatives of Blaschke products $\ldots \ldots \ldots \ldots \ldots \ldots \ldots \ldots \ldots$

Erhard Luft and Denis Karmen Sjerve, 3-manifolds with subgroups

$Z \oplus Z \oplus Z$ in their fundamental groups $\ldots \ldots \ldots \ldots \ldots \ldots \ldots \ldots \ldots \ldots \ldots \ldots$

George Clifford Nelson, Boolean powers, recursive models, and the Horn

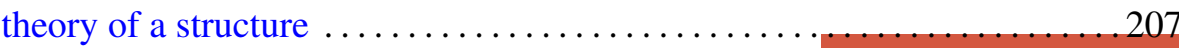

W. J. Phillips, Flow under a function and discrete decomposition of properly infinite $W^{*}$-algebras

Teodor C. Przymusiński, A solution to a problem of E. Michael ... 235

Bruce Harvey Wagner, Derivations of quasitriangular algebras 243 